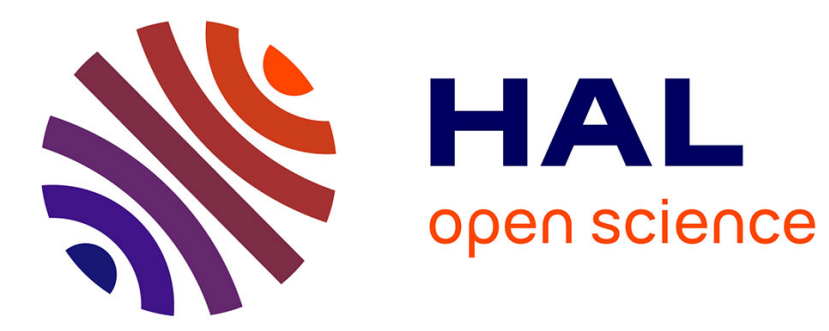

\title{
Sr2+-neutral molecules interactions: An assessment of theoretical procedures
}

Ane Eizaguirre, Manuel Yáñez, Jeanine Tortajada, Jean-Yves Salpin

\section{To cite this version:}

Ane Eizaguirre, Manuel Yáñez, Jeanine Tortajada, Jean-Yves Salpin. Sr2+-neutral molecules interactions: An assessment of theoretical procedures. Chemical Physics Letters, 2008, 464 (4-6), pp.240-244. 10.1016/j.cplett.2008.09.006 . hal-00368125

\section{HAL Id: hal-00368125 \\ https://hal.science/hal-00368125}

Submitted on 8 Oct 2018

HAL is a multi-disciplinary open access archive for the deposit and dissemination of scientific research documents, whether they are published or not. The documents may come from teaching and research institutions in France or abroad, or from public or private research centers.
L'archive ouverte pluridisciplinaire HAL, est destinée au dépôt et à la diffusion de documents scientifiques de niveau recherche, publiés ou non, émanant des établissements d'enseignement et de recherche français ou étrangers, des laboratoires publics ou privés. 


\title{
$\mathrm{Sr}^{2+}$-neutral molecules interactions. An Assessment of Theoretical Procedures.
}

Ane Eizaguirre ${ }^{\mathrm{a}}$, Manuel Yáñez ${ }^{\mathrm{a}}$, Jeanine Tortajada ${ }^{\mathrm{b}}$ and Jean-Yves Salpin ${ }^{\mathrm{b}}$

a Departamento de Química, C-9. Universidad Autónoma de Madrid. Cantoblanco. 28049Madrid. Spain.

${ }^{\mathrm{b}}$ Université d'Evry Val d'Essonne - Laboratoire d'Analyse et Modélisation pour la Biologie et l'Environnement

CNRS - UMR 8587 - Bâtiment Maupertuis, Boulevard François Mitterrand, 91025 Evry, France

\begin{abstract}
An assessment of different density functionals and basis sets in describing the binding of $\mathrm{Sr}^{2+}$ to bases which contain first and second row atoms as active sites has been performed. CCSD(T) calculations using a very large polarized QZ basis set were used as a reference. A total of 12 different functionals have been considered in our assessment. The G96LYP or O3LYP functionals when associated with an enlarged LANLDZ basis set for Sr, including $d$ and $f$ polarization functions and $s p$ diffuse functions, and a 6-311+G(3df,2p) basis set for the remaining atoms, yield $\mathrm{Sr}^{2+}$ binding energies in fairly good agreement with the $\operatorname{CCSD}(\mathrm{T}) / \mathrm{PQZ}$ reference calculations.
\end{abstract}




\section{Introduction}

The interest in the interactions between doubly charged metal ions and neutral bases in the gas phase has increased significantly in the last decade, among other things because the advent of electrospray ionization/mass spectrometry techniques provide a mild procedure to generate them [1]. On the other hand, the role of these interactions in many chemical and biochemical processes has been recognized for a long time [2]. It is well established, for instance, that the formation of polypeptides from amino acids is enhanced by the presence of metal dications [3], and that they play an important role in DNA bases pairing [4]. As a consequence many efforts have been devoted to the study of the structure and stability of complexes of metal dications with different biomolecules [4,5]. Metal dications are also assumed to be important in extreme environments, such as the upper planets atmospheres[6,7], and they can intervene in the generation of different astrochemical species [8]. Also importantly, the association of a doubly charged species to a neutral system is followed by a significant electron density rearrangements which often lead to unusual bonding properties [9-13]. We have paid particular attention in the last years to gas-phase reactions involving $\mathrm{Ca}^{2+}$ because, differently to what is usual when dealing with divalent transition metal ions, the $\mathrm{Ca}^{2+}$ complexes are thermochemically stable[14-17] and therefore the study of their unimolecular reactivity in the gas phase is feasible. In order to investigate the reactivity trends when moving down the group of alkaline-earth metals, we have initiated an investigation of gas-phase reactions involving $\mathrm{Sr}^{2+}$. These studies require a reliable description of the potential energy surfaces, which is usually attained through the use of $a b$ initio or density functional theory (DFT) calculations. A crucial point, however, is whether the standard approaches normally used in the study of neutral systems and monocations perform equally well when dealing with dications, and 
therefore, an assessment of the theoretical models available is needed. Some assessments have been reported for $\mathrm{Ca}^{2+}[18-20]$, but not for $\mathrm{Sr}^{2+}$. The main difficulty in these assessment studies is the complete lack of experimental information on the structures or binding energies of these species. Therefore, in our work we have used as a reliable reference, as far as binding energies are concerned, the results obtained using a CCSD(T) approach together with a very large basis set which will be described in detail in the next section. Since we are interested in the study of gas-phase reactions involving molecules of biochemical relevance, we have included in our assessment different DFT approaches, because they usually provide a good compromise between accuracy and computational cost.

\section{Computational Details}

Several effective core potentials accounting for relativistic effects have been proposed in the literature for Sr, which essentially differ in the number of basis set used to expand the valence shell of the atom [21]. The most extended one is that associated with the 10 -valence-electron pseudopotential proposed by Kaupp et al. [22]. This is a 7s5p4d basis on $\mathrm{Sr}$, and an all-electron $7 \mathrm{~s} 4 \mathrm{p} 3 \mathrm{~d} 2 \mathrm{f} 1 \mathrm{~g}$ basis set on first row atoms, such as $\mathrm{C}, \mathrm{O}$ and $\mathrm{N}$, and 9s6p4d2f1g on second row atoms, such as S and P [23]. Such a basis set becomes unpractical when dealing with very large molecular compounds, which is the final goal of our research, but is very adequate to get high accurate $\mathrm{Sr}^{2+}$ binding energies when used together with a $\operatorname{CCSD}(\mathrm{T})$ approach for small systems. Hence, this level, which will be named hereafter as $\operatorname{CCSD}(\mathrm{T}) / \mathrm{QZ}$, will be used as a reference in our assessment of the different functionals investigated. 
Eleven different functionals were analyzed, of which, nine are hybrid functionals. Three of them combine the Becke's three-parameters nonlocal hybrid exchange potential B3[24], with the LYP[25], P86[26], and PW91[27] correlation functionals. The other six are B972[28], MPW1PW91[29], PBE0, O3LYP[30], BHandH and BMK[31]. To these, three non-hybrid functionals: G96 [32], TPSS[33] and PBE[34] have been added. The former was used together with the LYP correlation functional and the other two with the TPSS [33] and PBE [34] correlation functionals, respectively.

Since we are interested in positively charged species, the first step of our assessment was focused in selecting the ECP that yields the best results for gas-phase basicities, acidities and ionization energies for systems for which this information is experimentally available. For this purpose we have considered the ionization energy and electron affinity of $\mathrm{Sr}$, the ionization energies of $\mathrm{Sr}_{2} \mathrm{O}$ and $\mathrm{SrF}$, the gas-phase acidity of $\mathrm{SrH}$, and the proton affinity and gas-phase basicity of $\mathrm{SrO}$ and $\mathrm{SrOH}$. Four ECP were considered in our survey, namely, CRENBL [35], SKBJC [36], Stuttgart [22] and LAND2DZ [23]. From the results obtained, which are summarized in Tables S1-S6 of the supplementary material, and although there is not a clear-cut preference between the different sets analyzed, we have chosen the LAND2DZ as one which provides, in average, results in reasonably good agreement with the experimental values.

\section{Optimization of the basis set for $\mathrm{Sr}$}

One of the limitations of the standard LANL2DZ basis is the absence of polarization functions which are important to describe correctly most molecular properties. Hence our first step to improve this basis set was to optimize a set of $d$ functions. For this purpose we used the $\mathrm{SrH}$ system, and the optimized value found was $\alpha_{d}=0.3$. Since our final goal is to 
get as accurate $\mathrm{Sr}^{2+}$ binding energies as possible, our next step was to extend the aforementioned basis set to get an expansion of similar quality to the $6-311+\mathrm{G}(3 \mathrm{df}, 2 \mathrm{p})$, which will be used for the remaining atoms of the system. Hence our strategy is similar to the one adopted previously for the optimization of extended basis sets for $\mathrm{Br}$, I [37] or $\mathrm{Sb}[38]$ and $\mathrm{Pb}$ [39]. The only difference with the aforementioned procedures is that we have used DFT methods, in particular the B3LYP approach, instead of ab initio formalisms.

To obtain the supplementary diffuse $s, p$ components and the $d, f$ polarization functions we have uncontracted the $\mathrm{p}$ valence of the LANL2DZ basis so it becomes formally a quadruple zeta expansion. To create multiple sets of $d$ functions from a single optimized function we have adopted the usual procedure, in which the new exponents are obtained as multiples, $n \alpha_{d}$, or fractions, $\alpha_{d} / n$, of the single optimized exponent $\alpha_{d}$. Using $\mathrm{SrH}$ as model systems, the aforementioned uncontracted scheme for $\mathrm{Sr}$ and a $311 \mathrm{G}(\mathrm{p})$ basis set for hydrogen, the best results were obtained for $n=3$. With the new basis so defined, the set of diffuse $s p$ functions (with the constraint $\alpha_{s}=\alpha_{p}=0.0093$ ), were optimized for the $\mathrm{SrH}^{-}$species, on its LANL2DZ optimized geometry. The set of $f$ polarization functions was optimized for $\mathrm{SrH}$, using the uncontracted basis for $\mathrm{Sr}$, supplemented with the $3 \mathrm{~d}$ polarization functions and using a $311 \mathrm{G}(\mathrm{p})$ basis for $H$. The value obtained was $\alpha_{\mathrm{f}}=0.08$. Hence, in what follows, geometry optimizations will be carried out using the LANL2DZ supplemented with one $d$ function and one set of $s p$ diffuse functions, whereas the remaining atoms are described using a $6-31+G(d, p)$ basis set. From now on, and for the sake of simplicity, we will refer to this basis set expansion as $6-31+G(d, p)$

Final energies will be obtained using for $\mathrm{Sr}$ the uncontracted LANL2DZ basis, supplemented with $3 d$ and $1 f$ polarization functions and a $s p$ diffuse component, and a 6- 
$311+\mathrm{G}(3 \mathrm{df}, 2 \mathrm{p})$ basis set expansion for the remaining atoms of the system. For the sake of simplicity this basis set will be named hereafter as $6-311+\mathrm{G}(3 \mathrm{df}, 2 \mathrm{p})$. In order to estimate the possible effect of the basis set superposition error (BSSE) in our assessment we have chosen as suitable model compounds water, as representative of the smaller systems and $\mathrm{CH}_{2} \mathrm{~S}$ and formamide as representative of the larger ones included in our study. The BSSE was calculated using the counterpoise method [40] at the $\operatorname{CCSD}(\mathrm{T}) / \mathrm{QZ}$ level and using the G96LYP functional, which is one of those that exhibit a better performance, as we shall discuss later. For the water complex, the BSSE at the CCSD(T)/QZ and G96LYP levels is 1.3 and $0.5 \mathrm{~kJ} \mathrm{~mol}^{-1}$, respectively. For $\mathrm{CH}_{2} \mathrm{~S}$ and formamide these values become 0.4 and $0.8 \mathrm{~kJ} \mathrm{~mol}^{-1}$, and 0.8 and $0.4 \mathrm{~kJ} \mathrm{~mol}^{-1}$, respectively. Hence, in all cases the BSSE can be considered rather small, and not significantly different when $\operatorname{CCSD}(\mathrm{T})$ ab initio or DFT approaches are used.

\section{Assessment of the functionals}

Bearing in mind that we are finally interested in the study of interactions involving different biochemical bases, we have defined a set of small molecules, namely water, formaldehyde, ammonia, methanimine, hydrogen cyanide, hydrogen sulfide, thioformaldehyde (methanethial), and phosphine, which contain as basic sites $\mathrm{O}\left(\mathrm{sp}^{3}\right)$, $\mathrm{O}\left(\mathrm{sp}^{2}\right), \mathrm{N}\left(\mathrm{sp}^{3}\right), \mathrm{N}\left(\mathrm{sp}^{2}\right), \mathrm{N}(\mathrm{sp}), \mathrm{S}\left(\mathrm{sp}^{3}\right), \mathrm{S}\left(\mathrm{sp}^{2}\right)$ and $\mathrm{P}\left(\mathrm{sp}^{3}\right)$. Since very often biomolecular bases contain several basic sites, favoring the formation of bridged structures in which the dication interacts with two or more basic sites simultaneously, we have added to the previous set formamide and hydroxylamine, as typical bidentate bases.

$\mathrm{Sr}^{2+}$ binding energies, $\mathrm{D}_{0}$, were evaluated by subtracting from the energy of the complex the energy of the neutral and that of $\mathrm{Sr}^{2+}$, after including the corresponding ZPE 
corrections. As indicated above, for all functionals, geometry optimizations and zero point vibrational energies were obtained using the $6-31+\mathrm{G}(\mathrm{d}, \mathrm{p})$ expansion defined above. Vibrational frequencies were evaluated using the harmonic approximation. Final energies were obtained in single point calculations using these optimized geometries and what we called a $6-311+\mathrm{G}(3 \mathrm{df}, 2 \mathrm{p})$ basis set.

A first level of assessment was carried out by comparing these results with those obtained at the CCSD(T)/6-311+G(3df,2p)//CCSD/6-31+G(d,p) level of theory.

To most accurate $\mathrm{Sr}^{2+}$ binding energies, which define our more reliable reference, were obtained at the $\operatorname{CCSD}(\mathrm{T}) / \mathrm{QZ} / / \mathrm{CCSD} / 6-31+\mathrm{G}(\mathrm{d}, \mathrm{p})$ level of theory.

All approaches provide rather similar geometries for the complexes investigated, which are not going to be discussed here. We report, however, in Figure S1 of the supplementary material, the values of the thermal corrections to the enthalpies, which, in principle are sensitive both to geometrical details and to the molecular force field. As can be seen, the values obtained do not differ significantly, either for first-row or second-row bases. Since, very often it is necessary to obtain free energies, we showed in Figure S2 of the supplementary material the entropy values obtained for first- and second-row bases. In general, the dispersion of the values obtained is very small, with the only exception of functionals TPSS, MPW1PW91 and BMK, which give too high entropy values for the complexes with phosphine.

As far as $\mathrm{Sr}^{2+}$ binding energies are concerned, we present in Figure 1 the results obtained in this assessment for ammonia, water and formaldehyde.

It can be seen that in general most of the functionals under investigation yield binding energies in reasonably good agreement with our $\operatorname{CCSD}(\mathrm{T}) / \mathrm{QZ}$ reference values, with perhaps the only exception of the $\mathrm{BHandH}$ functional which significantly 
overestimates the $\operatorname{CCSD}(\mathrm{T}) / \mathrm{QZ}$ values. The performance of $\mathrm{B} 3 \mathrm{LYP}$, one of the most popular hybrid functionals is reasonably good, but the best agreement is observed when the G96LYP and the O3LYP functionals are used. It is important to emphasized that the deviations of these two functionals are rather similar to those calculated at the CCSD(T)/6$311+\mathrm{G}(3 \mathrm{df}, 2 \mathrm{p})$ level $(\mathrm{CCSD}(\mathrm{T}) / \mathrm{A}$ in the figure caption).

When dealing with bases that contain a second-row atom as active site (see Figure 2), again the $\mathrm{BHandH}$ functional gives the largest deviations with respect to the $\operatorname{CCSD}(\mathrm{T}) / \mathrm{QZ}$ values, while the best performance is obtained for the G96LYP and the O3LYP functionals. However, although these two functionals yield quite small deviations for phosphine binding energies, they significantly overestimate the $\mathrm{SH}_{2}$ binding energy, and even more the thioformaldehyde binding energy. This overestimation is similarly big when using as a reference the $\operatorname{CCSD}(\mathrm{T}) / 6-311+\mathrm{G}(3 \mathrm{df}, 2 \mathrm{p})(\operatorname{CCSD}(\mathrm{T}) / \mathrm{A}$ in the figure) values instead of the more accurate $\operatorname{CCSD}(\mathrm{T}) / \mathrm{QZ}$ ones. It is worth noting that in these cases the other functionals also yield quite large deviations, with the only exception of the BMK functional, whose results are rather similar, for $\mathrm{S}$ and $\mathrm{P}$ bases to those obtained with the G96LYP and the O3LYP approaches.

The situation does not change significantly when considering the intrinsic basicities of $\mathrm{N}$ unsaturated bases, hydrogen cyanide and methanimine, with respect to $\mathrm{Sr}^{2+}$. As illustrated in Figure 3, once more the best performance is found for the G96LYP and the O3LYP hybrid functionals, and the worst agreement is attained when the BHandH functional is used. The case of formamide is rather interesting, because although the system presents two basic sites, only the structure in which the $\mathrm{Sr}^{2+}$ atom is attached to the oxygen atom is found to be a minimum of the potential energy surface. The $\mathrm{C}_{\mathrm{s}}$ structure, in which $\mathrm{Sr}^{2+}$ bridges between the carbonyl oxygen atom and the amino nitrogen (see Figure 4) is 
predicted to be a transition state no matter which functional is used for the geometry optimization. This structure is predicted to be a TS also at the CCSD/6-31+G(d,p) level.

It must be mentioned that the stability of this transition state is slightly underestimated when the G96LYP and the O3LYP functionals are used, whereas the best agreement in this case is found when the B3LYP functional is used.

For hydroxylamine, differently to what was found for formamide, all methods agree in predicting that the structure in which the metal dication is interacting with both basic sites (see Figure 4) is the global minimum of the potential energy surface, the oxygen bound complex being the second local minimum. No matter the method used, all attempts to obtain a complex in which $\mathrm{Sr}^{2+}$ is bound only to the amino group failed, because this arrangement collapses to the global minimum.

Again, in this case the performance of both G96LYP and O3LYP functionals is superior to the others, as illustrated in Figure 5. It is important to emphasize that all functionals investigated yield energy gaps between the two local minimum which are very similar and rather close to the $\operatorname{CCSD}(\mathrm{T}) / \mathrm{QZ}$ estimates.

The good performance of the O3LYP functional with respect to the popular B3LYP functional is in agreement with previous assessments reported in the literature, which concluded that the OPTX exchange functional is superior to the B88 one [30,41]. As a matter of fact, the O3LYP functional was found to outperform the B3LYP and other hybrid functionals for the evaluation of heats of reaction, and barrier heights for organic reactions [41], or in the calculations of the atomic exchange energies, total atomic energies, as well as in the predictions of ionization potentials, electron affinities, and proton affinities [42]. It was also found to be superior to B3LYP for predicting geometrical parameters of selected transition-metal compounds [43,44], or for describing metal-CO interactions [45]. The 
better performance of OPTX exchange functional included in the O3LYP approach is likely reflected in a better description of the electronic charge density, as it has been proved before in the literature [46] by comparing the electron density obtained with different functionals and that evaluated in full configuration interaction calculations. This comparison showed that the popular B3LYP functional was consistently outmatched by O3LYP [46].

Also a good performance of the G96LYP to describe metal-ligand and metal-metal bond energies simultaneously with main group atomization energies, atomic ionization potentials, and bond lengths was also reported previously in the literature [47]. This functional was also found to be quite well suited for the study of the binding of other alkaline-earth doubly charged cations $\left(\mathrm{Mg}^{2+}\right.$ and $\left.\mathrm{Ca}^{2+}\right)[19]$ as well as complexes involving $\mathrm{Pb}^{2+}[39]$.

Relative $\mathrm{Sr}^{2+}$ binding energies deserve also some attention. Our CCSD(T) calculations show that $\mathrm{Sr}^{2+}$ binding energies roughly follow the intrinsic basicity trends of the compounds under investigation, although some significant exceptions are also observed. Formaldehyde and $\mathrm{HCN}$ have almost identical proton affinities $\left(712.0\right.$ and $712.9 \mathrm{~kJ} \mathrm{~mol}^{-1}$, respectively) [48], but similarly to what was found for their $\mathrm{Ca}^{2+}$ binding energies [49], at the $\operatorname{CCSD}(\mathrm{T})$ level the former is predicted to bind $\mathrm{Sr}^{2+}$ stronger $\left(229.4 \mathrm{~kJ} \mathrm{~mol}^{-1}\right)$ than the latter $\left(218.4 \mathrm{~kJ} \mathrm{~mol}^{-1}\right)$. Both G96LYP and O3LYP lead to the same qualitative conclusion, although the binding energy predicted for formaldehyde is slightly overestimated (235 and $233 \mathrm{~kJ} \mathrm{~mol}^{-1}$, respectively). A similar situation is observed when ammonia and methanimine are compared. Their experimental proton affinities are almost identical (853.6 and $852.9 \mathrm{~kJ} \mathrm{~mol}^{-1}$, respectively) [48], while our $\operatorname{CCSD}(\mathrm{T})$ calculations predict for methanimine a $\mathrm{Sr}^{2+}$ binding energy $\left(240.1 \mathrm{~kJ} \mathrm{~mol}^{-1}\right)$ slightly larger than that of ammonia 
$\left(236.5 \mathrm{~kJ} \mathrm{~mol}^{-1}\right)$, as it was also found before for the corresponding $\mathrm{Be}^{2+}, \mathrm{Mg}^{2+}$ and $\mathrm{Ca}^{2+}$ binding energies [49]. Once more the same qualitative trend is predicted when the G96LYP and the O3LYP functionals are used, even though the agreement with the $\operatorname{CCSD}(\mathrm{T})$ values is better for the latter. Also interesting is the case of ammonia and formaldehyde. In this case ammonia has a proton affinity significantly larger than that of formaldehyde (see above); but quite unexpectedly their $\mathrm{Ca}^{2+}$ binding energies are reversed with respect to their proton affinities [49]. This is not the case however for the $\mathrm{Sr}^{2+}$ binding energies. Although the gap between $\mathrm{Sr}^{2+}$ binding energies for ammonia and formaldehyde $\left(7 \mathrm{~kJ} \mathrm{~mol}^{-}\right.$

$\left.{ }^{1}\right)$ is much smaller than the gap between their proton affinities $\left(142 \mathrm{~kJ} \mathrm{~mol}^{-1}\right)$, still ammonia is predicted to be a slightly stronger base than formaldehyde when the reference acid is $\mathrm{Sr}^{2+}$. The same trend is predicted when the O3LYP functional is used, although the gap is smaller $\left(3 \mathrm{~kJ} \mathrm{~mol}^{-1}\right)$, whereas the G96LYP predicts the $\mathrm{Sr}^{2+}$ binding energy for ammonia to be slightly smaller ( by $2 \mathrm{~kJ} \mathrm{~mol}^{-1}$ ) than that of formaldehyde.

Some interesting results are also observed when comparing the $\mathrm{Sr}^{2+}$ binding energies of water and formaldehyde with those of their sulphur containing analogues. $\mathrm{H}_{2} \mathrm{O}$ and $\mathrm{CH}_{2} \mathrm{O}$ are weaker bases than $\mathrm{SH}_{2}$ and $\mathrm{CH}_{2} \mathrm{~S}$ in protonation processes[48]. However, their $\operatorname{CCSD}(\mathrm{T}) \mathrm{Sr}^{2+}$ binding energies (201.4 and $229.4 \mathrm{~kJ} \mathrm{~mol}^{-1}$, respectively) are significantly larger than those of $\mathrm{SH}_{2}$ and $\mathrm{CH}_{2} \mathrm{~S}$ (142.2 and $171.8 \mathrm{~kJ} \mathrm{~mol}^{-1}$, respectively). This clearly indicates a preference of $\mathrm{Sr}^{2+}$ to bind oxygen rather than sulphur as has been already found for other alkaline-earth dications such as $\mathrm{Ca}^{2+}[50]$.

\section{Conclusions}

From our survey we can conclude that the G96LYP non-hybrid functional and the O3LYP hybrid functional, when used with a 6-311+G(3df,2p)-like basis set developed in 
this work, provide $\mathrm{Sr}^{2+}$ binding energies in very good agreement with those obtained at the $\operatorname{CCSD}(\mathrm{T}) / \mathrm{QZ}$ level. As a matter of fact, the average deviation for both functionals is $4 \mathrm{~kJ}$ $\mathrm{mol}^{-1}$, with a maximum deviation of 11.3 and $12.9 \mathrm{~kJ} \mathrm{~mol}^{-1}$, respectively which is found in both cases for the thioformaldehyde-Sr ${ }^{2+}$ complex. When the base presents more than one active site, such as formamide or hydroxylamine, the relative stability of the different local minima is also well reproduced.

Acknowledgements. This work has been partially supported by the DGI Project No. CTQ2006-08558/BQU, by the Project MADRISOLAR, Ref.: S-0505/PPQ/0225 of the Comunidad Autónoma de Madrid and by Consolider on Molecular Nanoscience CSD200700010. AE acknowledges a FPI grant from the Ministerio de Educación y Ciencia of Spain. A generous allocation of computing time at the CCC of the UAM is also acknowledged.

\section{References}

[1] P. Jayaweera, A. T. Blades, M. G. Ikonomou, P. Kebarle, J. Am. Chem. Soc. 112 (1990) 2452.

[2] D. Schröder, H. Schwarz, J. Phys. Chem. A 103 (1999) 7385.

[3] M. Remko, B. M. Rode, Chem. Phys. Lett. 316 (2000) 489.

[4] J. V. Burda, J. Sponer, J. Leszczynski, P. Hobza, J. Phys. Chem. B 101 (1997) 9670.

[5] J. Sponer, M. Sabat, J. V. Burda, J. Leszczynski, P. Hobza, J. Phys. Chem. B 103 (1999) 2528.

[6] J. M. C. Plane, M. Helmer, Faraday Discuss. 100 (1995) 411.

[7] O. Witasse, et al, Geophys. Res. Lett. 29 (2002) 1041.

[8] A. M. Lamsabhi, et al, J. Mass Spectrom. 43 (2008) 317.

[9] W. Koch, G. Frenking, J. Gauss, D. Cremer, J. Am. Chem. Soc. 108 (1986) 5808.

[10] M. W. Wong, L. Radom, Int. J. Mass Spectrom. Ion Processes 86 (1988) 319.

[11] Y. D. Hill, B. S. Freiser, C. W. Bauschlicher, J. Am. Chem. Soc. 113 (1991) 1507.

[12] D. Duflot, J.-M. Robbe, J.-P. Flament, J. Chem. Phys. 102 (1995) 355.

[13] J. Bertrán, L. Rodríguez-Santiago, M. Sodupe., J. Phys. Chem. B 103 (1999) 2310.

[14] I. Corral, O. Mó, M. Yáñez, J.-Y. Salpin, J. Tortajada, L. Radom, J. Phys. Chem. A 108 (2004) 10080.

[15] I. Corral, et al, Chem. Eur. J 12 (2006) 6787.

[16] C. Trujillo, O. Mó, M. Yáñez, J. Y. Salpin, J. Tortajada, ChemPhysChem 8 (2007) 1330. 
[17] C. Trujillo, O. Mó, M. Yáñez, J. Tortajada, J.-Y. Salpin, J. Phys. Chem. B 112 (2008) 5479.

[18] S. Petrie, L. Radom, Int. J. Mass Spectrom. 192 (1999) 173.

[19] M. Alcamí, A. I. González, O. Mó, M. Yáñez, Chem. Phys. Lett. 307 (1999) 244.

[20] I. Corral, O. Mó, M. Yáñez, A. Scott, L. Radom, J. Phys. Chem. A 107 (2003) 10456.

[21] K. L. Schuchardt, et al, J. Chem. Inf. Model. 47 (2007) 1045.

[22] M. Kaupp, P. V. Schleyer, H. Stoll, H. Preuss, J. Chem. Phys. 94 (1991) 1360.

[23] Basis Set Exchange (BSE) software and the EMSL Basis Set Library. https://bse.pnl.gov/bse/portal (2007).

[24] A. D. Becke, J. Chem. Phys. 98 (1993) 1372.

[25] C. Lee, W. Yang, R. G. Parr, Phys. Rev. B 37 (1988) 785.

[26] J. P. Perdew, Phys. Rev. B 33 (1986) 8822.

[27] J. P. Perdew, K. Burke, Y. Wang, Phys. Rev. B 54 (1996) 16533.

[28] P. J. Wilson, T. J. Bradley, D. J. Tozer, J. Chem. Phys. 115 (2001) 9233.

[29] C. Adamo, V. Barone, J. Chem. Phys. 108 (1998) 664.

[30] A. J. Cohen, N. C. Handy, Mol. Phys. 99 (2001) 607.

[31] A. D. Boese, J. M. L. Martin, J. Chem. Phys. 121 (2004) 3405.

[32] P. M. W. Gill, Mol. Phys. 89 (1996) 433.

[33] J. M. Tao, J. P. Perdew, V. N. Staroverov, G. E. Scuseria, Phys. Rev. Lett. 91 (2003).

[34] J. P. Perdew, K. Burke, M. Ernzerhof, Phys. Rev. Lett. 77 (1996) 3865.

[35] L. A. Lajohn, P. A. Christiansen, R. B. Ross, T. Atashroo, W. C. Ermler, J. Chem. Phys. 87 (1987) 2812.

[36] W. J. Stevens, M. Krauss, H. Basch, P. G. Jasien, Can. J. Chem. 70 (1992) 612.

[37] M. N. Glukhovtsev, A. Pross, M. P. McGrath, L. Radom, J. Chem. Phys. 103 (1995) 1878.

[38] A. I. González, O. Mó, M. Yáñez, J. Chem. Phys. 112 (2000) 2258.

[39] J.-Y. Salpin, J. Tortajada, M. Alcamí, O. Mó, M. Yáñez, Chem. Phys. Lett. 383 (2004) 561.

[40] S. F. Boys, F. Bernardi, Mol. Phys. 19 (1970) 553.

[41] J. Baker, P. Pulay, J. Chem. Phys. 117 (2002) 1441.

[42] X. Xu, W. A. Goddard, J. Phys. Chem. A 108 (2004) 8495.

[43] T. Strassner, M. A. Taige, J. Chem. Theo. Comput. 1 (2005) 848.

[44] A. Vargas, M. Zerara, E. Krausz, A. Hauser, L. M. L. Daku, J. Chem. Theo. Comput. 2 (2006) 1342.

[45] N. E. Schultz, B. F. Gherman, C. J. Cramer, D. G. Truhlar, J. Phys. Chem. B 110 (2006) 24030.

[46] A. D. Bochevarov, R. A. Friesner, J. Chem. Phys. 128 (2008).

[47] N. E. Schultz, Y. Zhao, D. G. Truhlar, J. Phys. Chem. A 109 (2005) 11127.

[48] NIST Chemistry Webbook. Standard Reference Database Number 69. Eds. P.J. Linstrom and W.G. Mallard, Release June 2005, National Institute of Standards and Technology, Gaithersburg MD, 20899 (http://webbook.nst.gov). (2005).

[49] I. Corral, O. Mó, M. Yáñez, L. Radom, J. Phys. Chem. A 109 (2005) 6735.

[50] A. M. Lamsabhi, O. Mó, M. Yáñez, R. J. Boyd, J. Chem Theor. Comput. 4 (2008) 1002. 


\section{Figure captions}

1. $\mathrm{Sr}^{2+}$ binding enthalpies (in $\mathrm{kJ} \mathrm{mol}^{-1}$ ) for water, ammonia and formaldehyde.

2. $\mathrm{Sr}^{2+}$ binding enthalpies (in $\mathrm{kJ} \mathrm{mol}^{-1}$ ) for phosphines, hydrogen sulfide and thioformaldehyde.

3. $\mathrm{Sr}^{2+}$ binding enthalpies (in $\mathrm{kJ} \mathrm{mol}^{-1}$ ) for hydrogen cyanide, methanimine and formamide. Formamide $(\mathrm{O})$ denotes the minimum in which $\mathrm{Sr}^{2+}$ is attached to the carbonyl oxygen. Formamide(N) denotes the TS in which $\mathrm{Sr}^{2+}$ bridges between $\mathrm{N}$ and $\mathrm{O}$.

4. $\mathrm{Sr}^{2+}$ complexes of formamide (a and b) and hydroxylamine (c and $\left.\mathbf{d}\right)$. Structure $\mathbf{a}$ is a transition state.

5. $\mathrm{Sr}^{2+}$ binding enthalpies (in $\mathrm{kJ} \mathrm{mol}^{-1}$ ) for hydroxylamine. $\mathrm{HONH}_{2}(\mathrm{O})$ denotes the minimum in which $\mathrm{Sr}^{2+}$ is attached to the oxygen atom $\mathrm{HONH}_{2}(\mathrm{~N})$ denotes the minimum in which $\mathrm{Sr}^{2+}$ bridges between $\mathrm{N}$ and $\mathrm{O}$. 